\title{
Eisen macht müde Mädchen munter
}

\begin{abstract}
Eine zwölfwöchige Eisensubstitution kann bei gebärfähigen Frauen Müdigkeitssymptome deutlich reduzieren - auch wenn sie nicht anämisch sind. Das ist das Ergebnis einer placebokontrollierten Studie aus der Schweiz.
\end{abstract}

\begin{abstract}
1-2\% der Patienten suchen ihren
Hausarzt wegen Müdigkeit auf, darunter dreimal so viel Frauen wie Männer. Die Frauen könnten möglicherweise von einer oralen Eisensubstitution profitieren. Dies ergab eine randomisierte kontrollierte Studie, in die 198 Französinnen zwischen 18 und 50 Jahren eingeschlossen wurden. Die Frauen waren nicht anämisch und ohne erkennbaren klinischen Grund müde. Sie hatten Hämoglobinwerte $\geq 12,0 \mathrm{~g} / \mathrm{dl}$, aber relativ niedrige Ferritinspiegel $(<50 \mu \mathrm{g} / \mathrm{l})$. Die Probandinnen erhielten zwölf Wochen lang täglich entweder $80 \mathrm{mg}$ Eisensulfat oder Placebo. Müdigkeit und Lebensqualität wurden zu Beginn und am Studienende mit verschiedenen Bewertungsskalen ermittelt.

In der Gruppe der Frauen, die eine Eisensupplementation erhalten hatten, er-
\end{abstract}

gab sich nach zwölf Wochen im Vergleich zur Kontrollgruppe ein Unterschied von 3,5 Punkten auf der Current and Past Psychological Scale, auf der die Müdigkeit bewertet wurde (CAPPS 0-40 Punkte: Eisengruppe -12,2, Placebo -8,7). Bei den Eisensupplementierten hatte sich die Müdigkeit um 48\% reduziert, während in der Kontrollgruppe ein Rückgang von $29 \%$ zu verzeichnen war. Auf Angst- und Depressionssymptome hatte die Supplementation keinen Einfluss.

Bereits nach sechs Wochen zeigten sich in der Eisengruppe auch Auswirkungen auf verschiedene Laborwerte: Das Hämoglobin war um $0,3 \mathrm{~g} / \mathrm{d}$ l gestiegen, Ferritin um $6,8 \mu \mathrm{g} / \mathrm{l}$, das mittlere korpuskuläre Volumen (MCV) um 1,2 fL, der Hämatokrit um $0,8 \%$, der Wert für den löslichen Transferrinrezeptor
(TfR) um $0,4 \mathrm{mg} / \mathrm{l}$ gefallen und die Transferrinsättigung (TfS) hatte sich um 6,6\% erhöht. Ähnliche Werte ergaben sich am Studienende. Am meisten profitierten Frauen von der Eisensupplementation, deren $\mathrm{Hb}$-Wert zu Studienbeginn unter $13,0 \mathrm{~g} / \mathrm{dl}$ lag.

Fazit: Der Hämoglobinwert dient als Anhaltspunkt, doch er spiegelt die Aktivität der Erythropoese nicht immer verlässlich wider. Die Autoren raten dazu, bei Frauen mit Müdigkeit ohne erkennbaren klinischen Grund und einem Ferritin unter $50 \mu \mathrm{g} / \mathrm{l}$ Eisen zu supplementieren, selbst dann, wenn das $\mathrm{Hb}$ über 12 $\mathrm{g} / \mathrm{dl}$ liegt. Der Effekt kann durch die Bestimmung der entsprechenden Blutmarker bereits sechs Wochen nach Therapiebeginn beurteilt und der Eisenmangel so bestätigt werden.

Dr. Christine Starostzik

Vaucher P. et al. Effect of iron supplementation on fatigue in nonanemic menstruating women with low ferritin: a randomized controlled trial. CMAJ 2012; online 9. Juli 2012; doi: 10.1503/ cmaj. 110950

\section{Verhaltenstherapie dämpft Hitzewallungen}

Nächtliche Schweißattacken und lästige Hitzewallungen - die meisten Frauen nach einer Brustkrebstherapie kennen dieses Problem. Britische Forscher testeten, ob eine kognitive Verhaltenstherapie die Beschwerden lindern kann.

D as Team vom Institut für Psychiatrie des King's College in London nahm in ihre aktuelle Studie knapp 100 Frauen auf, die unter mindestens zehn problematischen Hitzewallungen oder nächtlichen Schwitzattacken pro Woche litten. Alle hatten eine Brustkrebstherapie hinter sich und keine Hinweise auf weitere Läsionen.

Die Hälfte erhielt sechs Wochen lang je 90 Minuten pro Woche eine kognitive Verhaltenstherapie. Darin erfuhren sie, welche Faktoren die Menopausebeschwerden verstärken können (Stress, übertriebene Sorgen aufgrund der Symptome, ungünstige Schlafgewohnheiten). Die Psychologen arbeiteten mit ihnen Strategien aus, um solche Verstärker zu vermeiden, und trainierten Atemund Entspannungsübungen bei Hitze- wallungen. Schließlich erhielten die Frauen eine $\mathrm{CD}$ mit Anleitungen für das Training zu Hause. Die andere Hälfte bekam eine Standardnachsorge mit kurzen Informationen zu Optionen gegen Hitzewallungen. Nach neun und 26 Wochen wurden die Frauen nach Häufigkeit und Stärke der Hitzebeschwerden befragt und die Ergebnisse auf einer zehnstufigen Skala dargestellt, bei der ein Unterschied von zwei Punkten als klinisch relevant gilt.

$\mathrm{Zu}$ Beginn der Studie lagen die Werte bei 6,5 (Verhaltenstherapie) und 6,1 Punkten (Kontrollgruppe). Nach sechs Wochen Verhaltenstherapie war der Wert deutlich stärker zurückgegangen als in der Kontrollgruppe (um 3,1 vs. 1,1 Punkte). Insgesamt gingen die Beschwerden in der Gruppe mit Verhal- tenstherapie um $46 \%$ zurück, mit Standardnachsorge nur um 19\%. Die Unterschiede waren auch nach 26 Wochen, also mehrere Monate nach Therapieende, noch ähnlich groß: So waren die Menopausebeschwerden mit Verhaltenstherapie um $52 \%$, ohne nur um $25 \%$ verringert. Eine klinisch signifikante Reduktion der Beschwerden um zwei Punkte gelang mit Verhaltenstherapie nach neun Wochen ( 65 vs. $38 \%$ ) und nach 26 Wochen ( 78 vs. $33 \%$ ) ebenfalls deutlich häufiger. Zudem verbesserten sich mit Verhaltenstherapie auch Schlaf, Stimmung und Lebensqualität deutlich stärker.

Fazit: Die Forscher schließen daraus, dass eine kognitive Verhaltenstherapie bei Frauen nach einer Brustkrebsbehandlung durchaus helfen kann, typische Menopause-Symptome zu lindern.

Thomas Müller

Mann E et al. Cognitive behavioural treatment for women who have menopausal symptoms after breast cancer treatment (MENOS 1): a randomised controlled trial. Lancet Oncol. 2012;13(3):309-18 